\title{
Editorial
}

\section{RIP kinase 3 in necroptosis: does it take two or more to kill?}

\author{
C Kim ${ }^{1}$ and M Pasparakis*1 \\ Cell Death and Differentiation (2014) 21, 1505-1507; doi:10.1038/cdd.2014.100
}

Programmed cell death (PCD) has an important role in organismal physiology both during embryonic development and in adult tissue homeostasis. In addition to the longrecognized and well-studied caspase-dependent apoptosis, receptor-interacting protein kinase (RIPK)-mediated necrosis or necroptosis has been recently identified as a new type of programmed cell death with key functions during embryogenesis, tissue homeostasis and inflammation. ${ }^{1-8}$ Necroptosis is induced by multiple pathways, such as death receptors of the tumor necrosis factor receptor (TNFR) superfamily, TRIFdependent Toll-like receptor (TLR) signaling, and type I and type II interferon receptors. ${ }^{9}$ Necroptotic cell death is executed by RIPK3-mediated recruitment and phosphorylation of mixed lineage kinase domain-like protein (MLKL), ${ }^{10-12}$ which seems to kill cells by a mechanism dependent on its translocation to the plasma membrane. ${ }^{13-16}$ The activation of RIPK3 and recruitment of MLKL are thought to require the formation of a high-molecular weight signaling complex, termed the necrosome, which also contains RIPK1, caspase-8 and FADD. ${ }^{9}$ However, the stoichiometry of the necrosome complex and the mechanisms regulating its formation and activation remain only partly understood. FADD and caspase-8 negatively regulate necroptosis as inhibition of caspase-8 activity as well as knockdown or knockout of FADD or caspase-8 sensitize RIPK3-expressing cells to necroptosis. ${ }^{9}$ Studies based mainly on small molecule inhibitors of RIPK1, termed necrostatins, showed that inhibition of RIPK1 kinase activity blocks necroptosis in response to most stimuli, leading to the conclusion that RIPK1 kinase activity is essential for necroptosis. ${ }^{9,17}$ Also RIPK1-deficient mouse embryonic fibroblasts were protected from TNF-induced necroptosis, further supporting an essential role of RIPK1 acting upstream of RIPK3 to induce the formation and activation of the necrosome. ${ }^{9,17}$ These findings suggested that the RIP homotypic interaction motif (RHIM)-dependent formation of a RIPK1/RIPK3 heterodimer is critical for the induction of necroptosis. RHIM-dependent oligomerization of RIPK1 and RIPK3 was recently proposed to form a functional amyloid signaling complex triggering necroptosis. ${ }^{18}$ However, the stoichiometry, hierarchy and functional roles of RIPK1 and RIPK3 within the necrosome have remained elusive. Two studies reported in this issue of
'Cell Death and Differentiation' shed some light on this question.

In order to study how homodimeric or heterodimeric interactions between RIPK1 and RIPK3 regulate the assembly and activation of the necrosome, the Han and Oberst labs took advantage of chemically induced dimerization systems allowing to experimentally trigger RIPK1 and RIPK3 homo/ hetero-dimerization. ${ }^{19,20}$ They show that RIPK1 could only induce necroptosis upon RHIM-dependent recruitment of RIPK3, consistent with the notion that RIPK1/RIPK3 association is critical for necroptosis. However, surprisingly they found that RIPK1/RIPK3 heterodimers were not sufficient to trigger necroptosis unless additional RIPK3 molecules were recruited to the RIPK1/RIPK3 heterodimer via RHIM-dependent interactions. On the contrary, Orozco et al. showed that inhibition of RIPK1 kinase activity inhibited but RIPK1 deficiency potentiated necroptosis triggered by RIPK3 oligomerization, ${ }^{20}$ suggesting that RIPK1 acts as an inhibitor of necroptosis under certain conditions.

Wu et al. found that the formation of a RIPK3 homodimer was sufficient for the recruitment of MLKL and induction of necroptosis. ${ }^{19}$ Importantly, they showed that RIPK3 dimerization leads to intramolecular autophosphorylation of RIPK3, which is required to summon MLKL to induce necrotic cell death. These results identify the RIPK3 homodimer as the minimal basic unit that is sufficient for the recruitment and activation of MLKL and the induction of necroptosis. Using a different inducible RIPK3 dimerization system, Orozco et al. found that RIPK3 homodimers seed and propagate oligomerization of RIPK3 to induce necroptosis. Although the data from two studies largely concur with each other, there is one significant difference. Whereas $\mathrm{Wu}$ et al. ${ }^{19}$ clearly showed that the formation of a RIPK3 homodimer is sufficient to activate necroptosis, Orozco et al. ${ }^{20}$ found that RIPK3 homodimerization is not sufficient by itself to induce necrotic cell death but requires the recruitment of additional RIPK3 molecules to form oligomers to summon MLKL and trigger necroptosis. This discrepancy seems to be related to the RIPK3 domain used for the fusion of the dimerization interfaces. Wu et al. attached the dimerization domain to the $\mathrm{N}$-terminus of RIPK3 adjacent to its kinase domain, whereas Orozco et al. attached the dimerization domain at the

\footnotetext{
${ }^{1}$ Institute for Genetics, Centre for Molecular Medicine (CMMC) and Cologne Excellence Cluster on Cellular Stress Responses in Aging-Associated Diseases (CECAD), University of Cologne, 50931 Cologne, Germany

${ }^{*}$ Corresponding author: M Pasparakis, CECAD Research Center, University of Cologne, Joseph-Stelzmann-Strasse 26, D-50931 Cologne, Germany. Tel: +49 221478 84349; Fax: +49 221478 84350; E-mail: pasparakis@ uni-koeln.de
} 


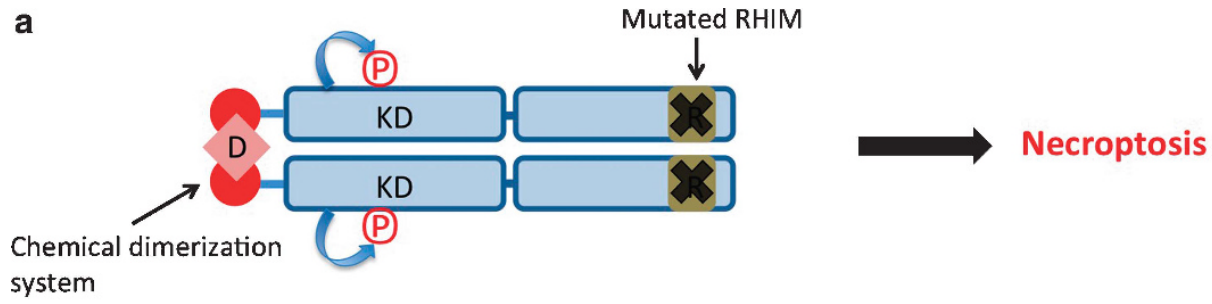

b

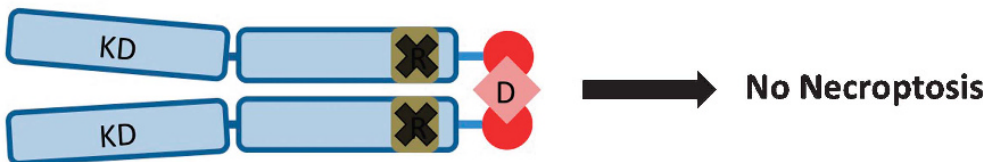

c

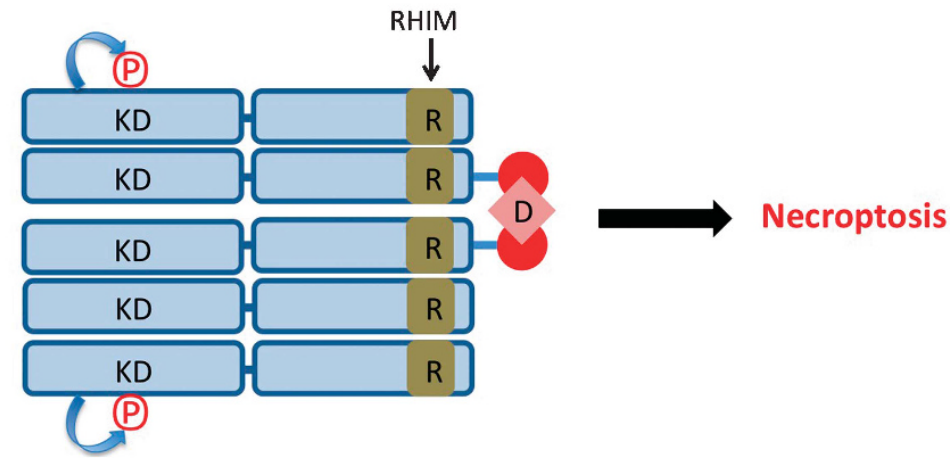

Figure 1 Schematic model of the differential effect of $\mathrm{N}$ - or C-terminal dimerization of RIPK3. (a) Chemical dimerization of RIPK3 by N-terminal fusion of the dimerization motifs is sufficient to induce autophosphorylation, MLKL recruitment and necroptosis. It is likely that N-terminal dimerization brings the kinase domains (KDs) of the two molecules in the dimer in close proximity inducing conformational changes resulting in kinase activation. (b) Chemical dimerization of RIPK3 by C-terminal fusion of the dimerization motifs is not sufficient to induce autophosphorylation and necroptosis. It is likely that C-terminal dimerization does not trigger the conformational changes required for kinase activation. (c) RHIM-dependent recruitment of additional RIPK3 molecules results in oligomerization and activation of RIPK3 autophosphorylation and necroptosis. $\mathrm{D}$, chemical dimerizer; R, RIP homotypic interaction motif (RHIM)

C-terminus, which is adjacent to the RHIM. In addition, when $\mathrm{Wu}$ et al. attached a different dimerization domain at the C-terminus of RIPK3 they also found that RIPK3 homodimers were not sufficient to induce cell death. It is therefore likely that $\mathrm{N}$ - or C-terminally enforced dimerization of RIPK3 results in different structural conformation of the molecules that affects their capacity to autophosphorylate and recruit MLKL (Figure 1). It is possible that $\mathrm{N}$-terminally fused dimerizers induce specific conformational changes to the adjacent kinase domains of the two RIPK3 molecules within the homodimer triggering their activation and autophosphorylation. The C-terminally fused dimerizers may not be capable of enforcing such conformational changes of the RIPK3 molecules to trigger kinase activation. In that case, the recruitment of additional RIPK3 molecules via RHIM-dependent interactions is required to induce kinase activity and autophosphorylation. Solving the structures of the dimeric and oligomeric RIPK3 complexes will be required to understand the molecular interactions triggering activation of the kinase and autophosphorylation.

Although the dimerization systems employed in these studies are undoubtedly invaluable tools to answer basic mechanistic questions on the minimal RIPK3 complexes that have the capacity to trigger necrosis, the artificial nature of these experiments leaves several questions open. Do RIPK3 homodimers exist in wild-type cells in physiologically relevant quantities, or do these molecules rapidly oligomerize under necroptosis-inducing conditions? Assuming that RIPK3 homodimers are prevalent and functionally relevant in wild-type cells, do they resemble the $\mathrm{N}$ - or the C-terminally enforced homodimers employed in these studies? As the RHIM domain is located close to the C-terminus of RIPK3, it is rational to postulate that placing the dimerizer at the C-terminus might resemble more the $\mathrm{RHIM}$-dependent interaction, as Oberst and colleagues argue. However, $\mathrm{RHIM}$-dependent interactions may trigger very different conformational changes to the molecules compared with the chemical dimerizer; therefore, this hypothesis awaits experimental validation from structural studies. $\mathrm{Be}$ it as it may, the studies by the Han and Oberst labs provide new and important information on which subsequent work will build on in order to unravel the molecular mechanisms regulating this new and fascinating pathway to cell death.

\section{Conflict of Interest}

The authors declare no conflict of interest.

1. Oberst A et al. Nature 2011; 471: 363-367.

2. Kaiser WJ et al. Nature 2011; 471: 368-372.

3. Zhang $\mathrm{H}$ et al. Nature 2011; 471: 373-376.

4. Bonnet MC et al. Immunity 2011; 35: 572-582.

5. Welz PS et al. Nature 2011; 477: 330-334. 
6. Dillon CP et al. Cell Rep 2012; 1: 401-407.

7. Duprez L et al. Immunity 2011; 35: 908-918.

8. Upton JW, Kaiser WJ, Mocarski ES. Cell Host Microbe 2010; 7: 302-313.

9. Vanden Berghe T et al. 2014; 15: 135-147.

10. Sun L et al. Cell 2012; 148: 213-227.

11. Zhao J et al. Proc Natl Acad Sci USA 2012; 109: 5322-5327.

12. Murphy JM et al. Immunity 2013; 39: 443-453.

13. Cai $Z$ et al. Nat Cell Biol 2014; 16: 55-65.
14. Chen $X$ et al. Cell Res 2014; 24: 105-121.

15. Dondelinger $Y$ et al. Cell Rep 2014; 7 : 971-981.

16. Wang $\mathrm{H}$ et al. Mol Cell 2014; 54: 133-146.

17. Christofferson DE, Li Y, Yuan J. Annu Rev Physiol 2014; 76: 129-150.

18. Li J et al. Cell 2012; 150: 339-350.

19. Wu X-N et al. Cell Death Differ 2014; e-pub ahead of print 6 June 2014; doi:10.1038/ cdd.2014.77.

20. Orozco S et al. Cell Death Differ 2014; 21: 1511-1521. 